\title{
Thermal extrusion of a non-linear viscoelastic solid inside a long cylindrical tube*
}

\author{
J. M. Kramer, J. D. Demaree** and E. E. Gruber \\ Reactor Analysis and Safety Division, Argonne National Laboratory, Argonne, IL 60439, USA
}

\begin{abstract}
An analysis is presented for the axial extrusion of an expanding viscoelastic solid inside a long cylindrical tube. Both differential thermal expansion and expansion of gas bubbles in the solid are considered as driving mechanisms. Finite element calculations are used to illustrate the details of the behavior of the system. The response is shown to be initially elastic followed by the development of a boundary layer near the free surface where the pressure gradients are sufficiently large to cause axial viscous flow. Simple boundary layer equations are derived to describe this flow. Results using these equations agree well with the results of the finite element calculations. The theory is applied to the extrusion of uranium-based metallic reactor fuel during overheating transients.
\end{abstract}

\section{Introduction}

Extrusion of an expanding viscoelastic solid inside a long cylindrical tube is of general engineering interest in forming and processing of metals, polymers and food products. Our application stems from renewed interest (Walters, Seidel and Kittel 1984) in uranium-based metal alloys as a fuel for sodium-cooled fast reactors. Here expansion and subsequent axial extrusion of overheated fuel has been postulated (Cahalan, Sevy and Su 1985; Miles and Kalimullah 1985) to provide a passive reactivity feedback mechanism which could contribute to reactor shutdown during an unprotected accident event. During such an event, the temperature of the fuel would rise due to the power-to-flow mismatch causing the fuel to expand by thermal expansion and by expansion of the fission gases generated in the fuel during irradiation. The fuel is enclosed in cylindrical cladding tubes so that it is free to expand only in the axial direction. If this expansion were uniform throughout the core it would provide a very strong negative reactivity feedback of approximately $10 \$ / \mathrm{mm}^{1}$.

Of course even if a solid such as metallic reactor fuel has a considerable potential for unrestrained swelling when heated, this swelling may not translate to significant axial extrusion when it is restrained within a long cylindrical tube. Hydrostatic pressure will develop in the tube to counteract the expansion, and large shear deformations are required to relieve the pressure and to move the material axially. This motion is limited by the magnitude of the pressure gradients and by the effective viscosity so that much of the extrusion may only occur near the free surface at the top of the fuel.

In the present paper we consider the general problem of the thermally driven extrusion of nonlinear viscoelastic solid inside of a long cylindrical tube. In the first section we give a brief discussion of the purely elastic response and compare generalized plane strain results with finite element calculations for the boundary layer near the free surface of the solid slug. In subsequent sections we consider more general non-linear viscoelastic behavior and show how the boundary layer grows

* Work supported by the U.S. Department of Energy, Office of Technology Support Programs under Contract W-31-109-Eng-38

** Presently attending the University of Michigan

1 Reactive $\varrho$ is a measure of excess neutrons. When $\varrho$ is divided by the delayed neutron fraction the ratio is termed reactivity in 'dollars'. The significance of fuel expansion in current fast reactor designs can be seen by noting that $1 \mathrm{~mm}$ of uniform expansion would balance the positive reactivity feedback of $10 \$$ resulting from the accidental withdrawl of one of the control rods 
downward as the solid begins to flow: Finally, approximate expressions are derived for the viscous flow limits and for the boundary layer penetration, and the results are applied to analyze the extrusion of metallic reactor fuel under transient heating conditions.

\section{Elastic behavior}

Consider a solid slug if length $L$ inside a long cylindrical tube of radius $R$ as shown in Fig. 1. It is assumed that the slug is firmly attached to the inside of the cylinder. For our application to metallic reactor fuel, the fuel is metallurgically bonded to its cladding after several atom percent burn-up. Typical ratios of $L / R$ for fast reactor fuel are 180 for Experimental Breeder Reactor-II (EBR-II) pins and 295 for proposed commercial reactor designs.

Now imagine the slug, designated by the subscript $F$ (fuel), and the cylinder, designated by the subscript $\mathrm{C}$ (cladding), heated at a rate $\dot{T}$. For simplicity we neglect the internal and external pressure and assume that the heating is uniform and that the elastic properties are constant as given in Table 1. Because the effective coefficient of thermal expansion of the slug is greater than the cylinder, the slug goes into a state of compression while the cylinder goes into a state of tension. A common

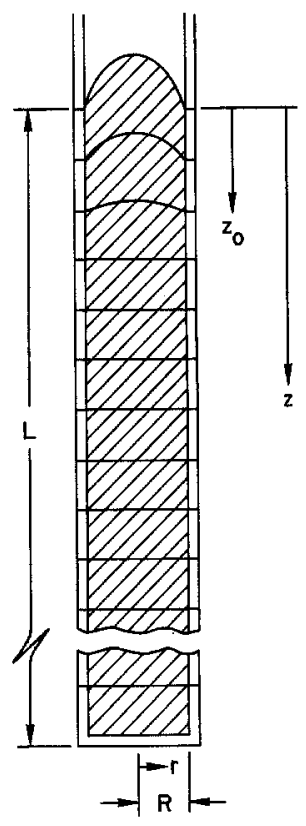

Fig. 1. Extrusion geometry showing the fuel slug (crosshatched) extruding inside its cladding tube. Relatively motion between the fuel and the cladding has developed over a boundary layer of length $z_{0}$

Table 1. Nominal material properties and dimensions used in calculations

\begin{tabular}{|c|c|c|c|c|c|c|c|c|c|}
\hline \multicolumn{10}{|c|}{ Properties } \\
\hline \multirow[t]{2}{*}{ Case } & \multirow[t]{2}{*}{$L / R$} & \multicolumn{2}{|c|}{$\begin{array}{l}\text { Elastic modulus, } \\
\text { Gpa }\end{array}$} & \multicolumn{2}{|c|}{ Poisson's ratio } & \multicolumn{2}{|c|}{$\begin{array}{l}\text { Linear expansion } \\
\text { coefficient, } 1 / K\end{array}$} & \multirow{2}{*}{$\begin{array}{l}\text { Creep } \\
\text { power } \\
n\end{array}$} & \multirow{2}{*}{$\begin{array}{l}\text { Creep coefficient, } \\
\mathrm{MPa}^{-n} \mathrm{~s}^{-1} \\
C_{\mathrm{F}}\end{array}$} \\
\hline & & $E_{\mathrm{F}}$ & $E_{\mathrm{C}}$ & $v_{\mathrm{F}}$ & $v_{\mathrm{C}}$ & $\alpha_{F}$ & $\alpha_{C}$ & & \\
\hline E1 & 3.2 & 14 & 125 & 0.23 & 0.33 & $2.1 \times 10^{-5}$ & $1.9 \times 10^{-5}$ & - & - \\
\hline F1 & 3.2 & 14 & 125 & 0.23 & 0.33 & $2.1 \times 10^{-5}$ & $1.9 \times 10^{-5}$ & Eqs: (5) & Eqs: (5) \\
\hline $\mathrm{F} 2$ & 6.4 & 14 & 125 & 0.23 & 0.33 & $2.1 \times 10^{-5}$ & $1.9 \times 10^{-5}$ & Eqs. (5) & Eqs. (5) \\
\hline L1 & 6.4 & 14 & 1250 & 0.23 & 0.33 & $2.1 \times 10^{-5}$ & $1.9 \times 10^{-5}$ & 1 & $3.33 \times 10^{-6}$ \\
\hline $\mathrm{L} 2$ & 6.4 & 14 & 1250 & 0.23 & 0.33 & $2.1 \times 10^{-5}$ & $1.9 \times 10^{-5}$ & 1 & $3.33 \times 10^{-7}$ \\
\hline S1 & 6.4 & 14 & 1250 & 0.23 & 0.33 & $3.9 \times 10^{-5}$ & $1.9 \times 10^{-5}$ & 1 & $3.33 \times 10^{-7}$ \\
\hline
\end{tabular}

Dimensions (for all cases); inner cylinder radius $R=1.91 \mathrm{~mm}$; cylinder thickness $h=0.30 \mathrm{~mm}$ 
misconception in reactor fuel pin analysis is that a uniform shear stress also develops along the length $L$ where the fuel and cladding are bonded together. This is not the case as can easily be seen by imagining the fuel removed from the cladding, allowing each to expand freely, and then calculating the uniform interface pressure and the uniaxial stress in the fuel and in the cladding so that both have the same axial strain and the same radial displacement at the interface and so that the total force is zero in the axial direction. The resulting simple stress state satisfies all of the equations and all of the boundary conditions except at the free surface $z=0$. There the surface integral of the axial stress $\sigma_{z}$ across both the slug and the cylinder is zero rather than $\sigma_{z}(r)=0$ for all $r$.

The simple solution outlined above is, of course, just the solution to the problem of the axisymmetric differential expansion of two infinitely long cylinders (Gatewood 1957) where the generalized plane strain assumption that the axial strain $\varepsilon_{z}$ is independent of $r$ is appropriate. Such an assumption has commonly been made in analyzing the mechanical response of nuclear fuel pins, although there have been some notable exceptions (Matthews 1970; Valentin and Carey 1970) where the stresses in stacks of short $(L / R \sim 2)$ ceramic fuel pellets were studied. For large $L / R$ ratios of interest here, however, Saint Venant's principle suggests that the generalized plane strain solution should be valid sufficiently far from the free surface. Near the upper boundary the difference between the actual stress state and the stress state for infinite cylinders decays (Toupin 1965) over a short boundary layer.

As a prelude to the desired calculation of viscoelastic behavior, a series of thermal-elastic finite element calculations were performed for cylinders with various $L / R$ ratios. The STRAW finite element code (Schreyer, Kennedy and Schoeberle 1983) was employed for these calculations using 5 equiaxed continuum elements through the solid slug and 4 continuum elements through the cylindrical tube. A segment of tube about one radius in length was extended above the upper interface. The length $L$ of the slug for each geometry was constructed by adding additional rows of elements. Obviously such a procedure could result in very long computation times if it were necessary to model an entire slug with $L / R$ ratios of 180 or 295 , as would be the case for fast reactor fuel pins. However, for the purposes of this paper a maximum $L / R$ ratio of 6.4 was sufficient to determine the extent of the boundary layer and to investigate how extrusion occurs as flow develops.

Figures 2, 3, and 4 show typical thermal-elastic results for $L / R=3.2$ when the slug and cylinder are heated $600 \mathrm{~K}$. The properties and dimensions that were assumed are given under case E1 in Table 1. As shown in Fig. 2, the axial stress $\sigma_{z}$ in the slug varies from zero at the upper surface to a nearly constant negative value at distances greater than about $2.5 R$. At the same distances Fig. 3 shows that the shear stress $\sigma_{r z}$ rapidly decreases to zero. Nearly identical stresses are calculated for greater $L / R$ ratios confirming that the elastic boundary layer is confined to the region adjacent to the free surface.

The displacements of the top 6 rows of elements shown in Fig. 4 illustrate the transition to generalized plane strain with $\varepsilon_{z}$ becoming independent of $r$ for elements at increasing distances from the free surface. The asymptotic values at large $z$ for the stresses and displacements in the slug can easily be determined from the generalized plane strain solution (Gatewood 1957). Substitution of the properties and dimensions for case E1 of Table 1 into the appropriate equations gives $\sigma_{z}=-17.6$ $\mathrm{MPa}, \sigma_{r}=-13.6 \mathrm{MPa}$ and $\varepsilon_{z}=1.18 \times 10^{-2}$. The constant value of $\sigma_{z}$ across the radius of the slug is in agreement with the limit of the finite element calculations shown in Fig. 2 at distances greater than $2.5 R$. Comparison of the finite element displacements shown in Fig. 4 with the generalized plane strain solution can be made by noting that for $L / R=3.2$ the distance from the bottom of the slug to the last row of elements is $2 R=3.82 \mathrm{~mm}$. For $\varepsilon_{z}=1.18 \times 10^{-2}$ the uniform displacement along the bottom of the last row should equal $45.1 \mu \mathrm{m}$, in agreement with the shown results.

\section{Viscoelastic constitutive equations}

Of primary interest in this paper are conditions where the solid slug shown in Fig. 1 is able to flow plastically and extrude inside the tube when both are heated. We will continue to assume, however, that the tube behaves thermo-elastically. In this section we outline the assumed deformation behavior of the slug. For convenience the subscript $\mathrm{F}$ is dropped, components of all tensors are given in a rectangular cartesian coordinate system, summation notation is used and the equations are written in their form for small deformations. 

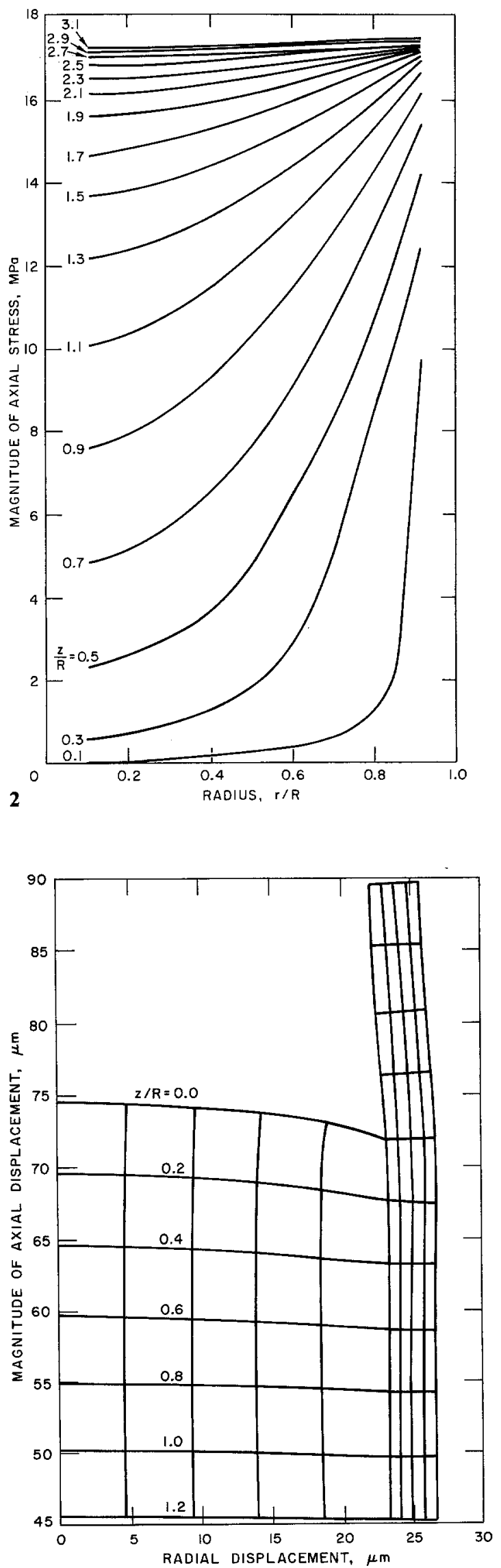

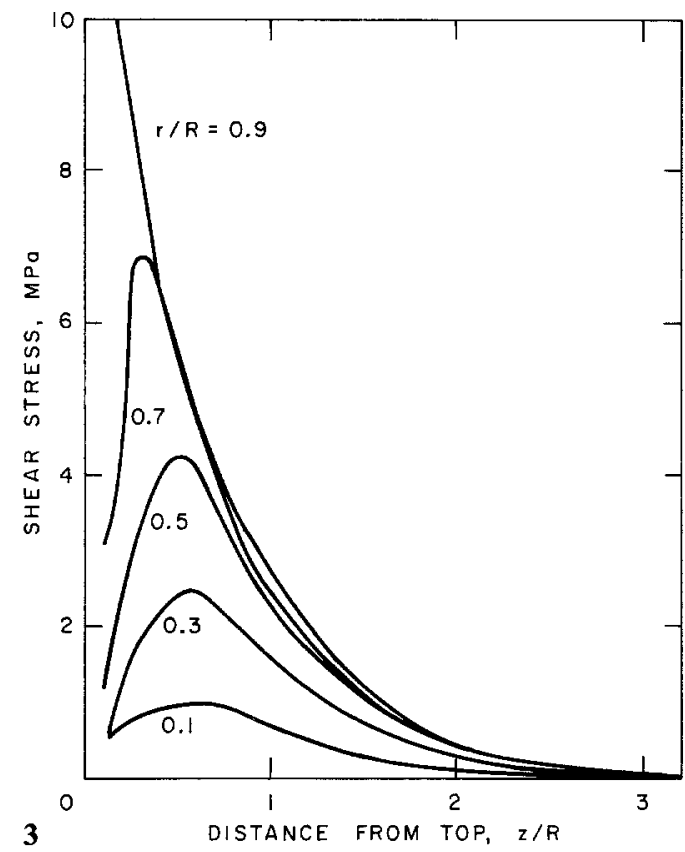

Figs. 2 and 3. 2 Magnitude of axial stress in an elastic solid fuel slug near the free surface. The different curves are for various normalized distances from the surface. 3 Shear stresses in an elastic solid fuel slug near the free surface. The different curves are for various normalized distances from the centerline
Fig. 4. Magnitude of axial and radial displacement at nodal points for an elastic fuel slug and an elastic cladding tube 
It is assumed that the total strain rate $\dot{\varepsilon}_{i j}$ can be broken into a deviatoric part $\dot{e}_{i j}$ and a volumetric part $\dot{e}_{m}$. The deviatoric component is given as the sum of the elastic strain rate $\dot{e}_{i j}^{\mathrm{e}}$ and the plastic strain rate $\dot{e}_{i j}^{\mathrm{p}}$, or

$\dot{e}_{i j}=\dot{e}_{i j}^{\mathrm{e}}+\dot{e}_{i j}^{\mathrm{p}}$,

where the dot stands for differentiation with respect to time $t$. For isotropic elastic behavior the deviatoric part of the elastic strain rate is related to the deviatoric stress rate $\dot{S}_{i j}$ by

$\dot{e}_{i j}^{\mathrm{e}}=\dot{S}_{i j} / 2 G$

where $G=E / 2(1+v)$ is the shear modulus.

We further assume that the plastic deformation can be determined from the von Mises flow function and associated flow rule. The effective stress $\sigma$ is then related to the effective plastic strain rate $\dot{\mathrm{e}}^{\mathrm{p}}$ by a material function which is chosen here to be of the non-linear power-law form

$\dot{\mathbf{e}}^{\mathrm{p}}=C(T) \boldsymbol{\sigma}^{n}$

where $C$ and $n$ are material properties and $T$ is the temperature. Individual components of the plastic strain rate are given by the associated flow rule

$\dot{e}_{i j}^{\mathrm{p}}=\frac{3}{2} \frac{\dot{\mathrm{e}}^{\mathrm{p}}}{\boldsymbol{\sigma}} S_{i j}$

For the uranium alloys and conditions of interest in this paper the power law parameters $C$ and $n$ are determined from the creep equations (Miles and Kalimullah 1985)

$\dot{\mathrm{e}}^{\mathrm{p}}=\left(5000 \sigma+6.0 \sigma^{4.5}\right) \exp (-26,170 / T)$

for $T<923 \mathrm{~K}$ and

$\dot{\mathbf{e}}^{\mathrm{p}}=0.08 \boldsymbol{\sigma}^{3} \exp (-14,340 / T)$

for $923 \mathrm{~K} \leq T \leq T_{m}$, where $\sigma$ is in $\mathrm{MPa}, \dot{\mathrm{e}}^{\mathrm{p}}$ is in $\mathrm{s}^{-1}, T_{m}$ is the solidus temperature (approximately $1373 \mathrm{~K}$ ), and $923 \mathrm{~K}$ represents the temperature of the transition to the solid solution $\gamma$ phase where there is a marked increase in the creep rate.

It is usual to assume that viscoelastic materials are plastically incompressible. The volumetric strain rate is then determined by the elastic compressibility and the thermal expansion, or

$\dot{e}_{m}=\frac{1}{v} \frac{\mathrm{d} v}{\mathrm{~d} t}=\frac{\dot{\sigma}_{m}}{K}+3 \alpha \dot{T}$

where

$K=\frac{E}{3(1-2 v)}$

is the bulk modulus, $\dot{e}_{m}=\dot{\varepsilon}_{i i}, \dot{\sigma}_{m}=\frac{1}{3} \dot{\sigma}_{i i}$ and $v$ is the specific volume. In our application to metallic nuclear fuels we are also interested in swelling due to retained fission gas. We will assume here that the gas is contained in small equilibrium bubbles that are bound by the surface tension. As shown in the Appendix, this type of swelling can be combined with the thermal expansion to give an effective linear expansion coefficient $\alpha$. Furthermore, if the bubbles are so large that their compressibility is important, the bubble compressibility can be added to the elastic compressibility $(1 / K)$ to give an effective bulk modulus.

For short times and high heating rates the viscoelastic model given above would be expected to result in elastic behavior. At the other extreme of long times and low heating rates it is reasonable to expect that the plastic strains will dominate the elastic strains and that the slug will begin to flow like a fluid with the normal stresses tending to a hydrostatic limit and with the velocity gradient in the radial direction dominating the strain-rate tensor. In anticipation of this result we note from the above equations that if $\sigma_{r}=\sigma_{\theta}=\sigma_{z}=-P$ and $\partial v_{z} / \partial r \gg \partial v_{r} / \partial z$

$\sigma=\sqrt{3} \sigma_{r z}$ 
$\dot{\mathrm{e}}^{\mathrm{p}}=\frac{2}{\sqrt{3}} \dot{e}_{r z}^{\mathrm{p}}=\frac{1}{\sqrt{3}} \frac{\partial v_{z}}{\partial r}$,

where $v_{r}$ and $v_{z}$ are the radial and axial components of the velocity. Dividing Eq. (8) by Eq. (9) shows that the effective viscosity $\eta$ is related to the material law given in Eq. (3) by

$\eta \equiv \frac{\sigma_{r z}}{\mathrm{~d} v_{z} / \mathrm{d} r}=\sigma / 3 \dot{\boldsymbol{\varepsilon}}^{\mathrm{p}}=\frac{\sigma^{1-n}}{3 C}$.

It should be noted that the effective viscosity of the solid material as calculated using this equation and Eq. (5) is some 10-15 orders of magnitude greater than the viscosity of liquid fuel (Ofte 1967) at the solidus.

\section{Viscoelastic behavior}

We return now to the consideration of the thermal extrusion of a viscoelastic solid within a long cylindrical tube. It has already been shown that for elastic behavior any relative motion between the centerline of the solid slug and the cylinder is limited to a small boundary layer near the free surface as seen in Fig. 4. Of interest here is how that boundary layer grows and how extrusion develops as the viscoelastic slug begins to flow. This behavior will be illustrated by repeating the previous calculations using the constitutive equations for plastic flow given in the last section.

The implicit version of the STRAW code was again used for the finite element calculations. Implicit time integration is performed in STRAW by the Newmark $\beta$-method with the value of $\beta$ $=1 / 4$, corresponding to trapezoidal integration. For this value of $\beta$, the method is unconditionally stable, and the time step is limited principally by accuracy, which is dictated by the modified Newton procedure used to solve the non-linear finite element equations. Iterations are performed until the error in energy during any time step is small compared to the total energy. Convergence of the procedure for the geometry and time scales of interest in this paper was verified by comparing STRAW code results with the analytical solution for the uniaxial creep of an unrestrained fuel slug with an instantaneously applied axial stress.

The problem we wish to consider is a metallic fuel pin, shown in Fig. 1, undergoing a power transient such that the temperature increases from a steady-state temperature of $773 \mathrm{~K}$ to melting $(1373 \mathrm{~K})$ at a heating rate of $0.1 \mathrm{~K} / \mathrm{s}$. Figure 5 shows the calculated displacements of the upper 6 rows of elements at the end of $6000 \mathrm{~s}$. The $L / R$ ratio for these calculations was 3.2 and the properties are

given under Case F1 in Table 1. The temperature increase was $600 \mathrm{~K}$ so that the results can be compared directly with results of the elastic calculations shown in Fig. 4. It is obvious that significant relative flow between the slug and the cylinder has been developed and that the relaxation of the stresses has caused elastic recovery in the cylinder.

Figure 6 shows the time history of the axial displacement of the free surface at $r=0$ for $L / R=3.2$ and for a second calculation with $L / R=6.4$ (Case F2). The initial response is elastic and can be calculated approximately from the generalized plane strain solution, as shown by the lower asymptote in Fig. 6. Significant plastic flow does not begin until times greater than $1500 \mathrm{~s}$ where the cubic creep law in Eq. (5), for the solid solution phase, is applicable.

A detailed study of the results from the above calculations indicates the following sequence of events leading to extrusion: 1) the initial response is nearly elastic and can be determined by generalized plane strain calculations; 2) as creep begins the stresses at any axial elevation relax to a hydrostatic state, again through plastic deformation; 3) the resulting axial pressure gradients are small except near the free surface where shear deformation begins; 4 ) as the shear deformation relieves the pressure gradient, a boundary layer (labeled $z_{0}$ in Fig. 1) separating flowing material from the rest of the slug moves downward until it reaches the bottom of the cylinder; 5) when the boundary layer reaches the bottom of the cylinder the entire slug flows like a fluid at a constant rate as indicated by the asymptote labeled 'viscous response' in Fig. 6. Obviously it takes a longer time for the flow to fully develop for the longer cylinder as shown in the figure.

As a measure of the boundary layer penetration in the finite element calculations we have plotted for each elevation the time history of the ratio of the axial displacement at the cladding interface to the 

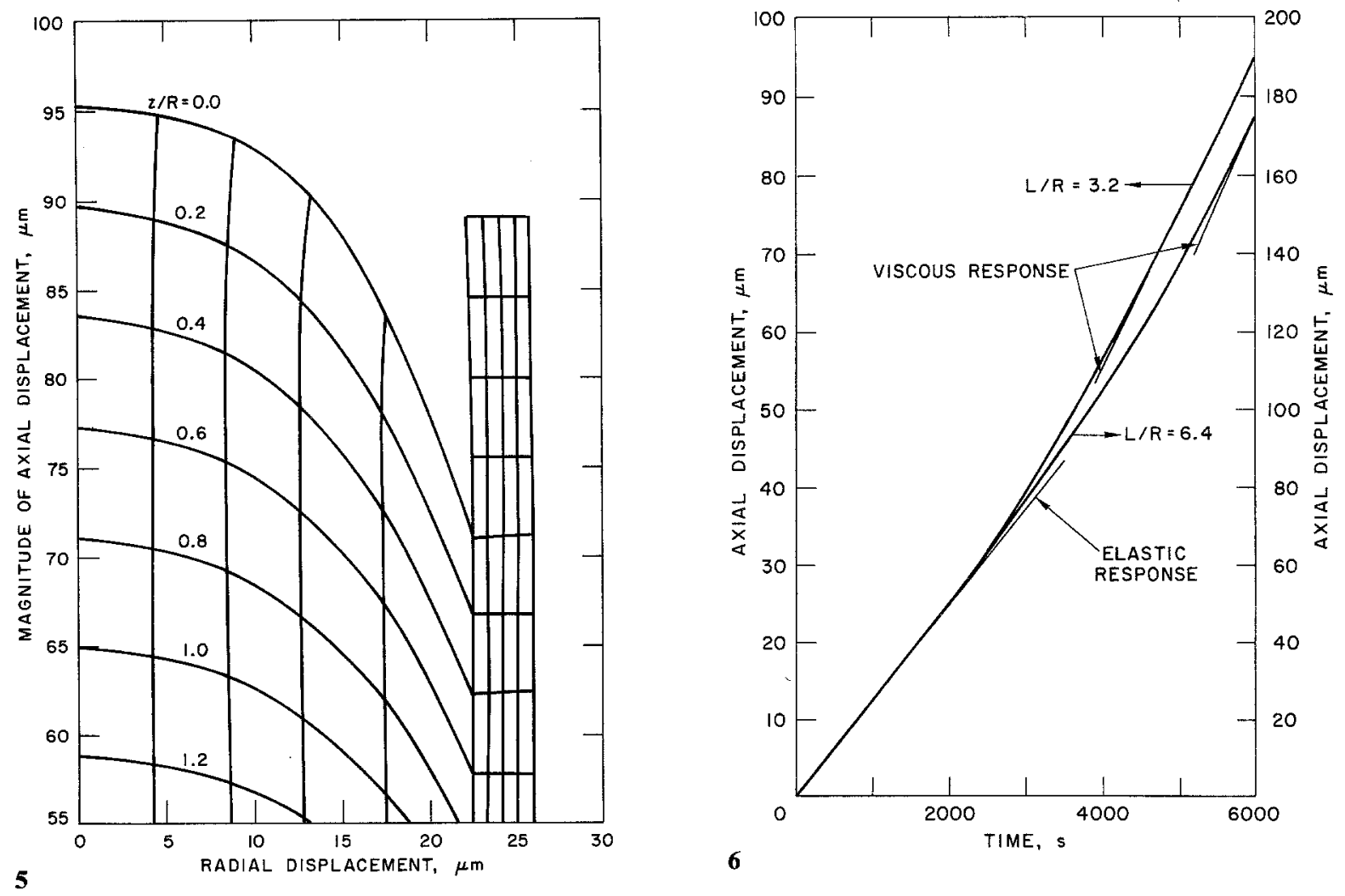

Figs. 5 and 6. 5 Magnitude of axial and radial displacements at nodal points for a viscoelastic fuel slug and an elastic cladding tube. 6 Magnitude of axial displacement at the centerline of the free surface for extrusion of short visoelastic fuel slugs with $L / R$ ratios of 3.2 and 6.4

axial displacement at the centerline. For elevations undergoing plane deformation this ratio remains near 1.0. The location of the boundary layer at any given time was then defined as the location above in which the ratio was less than some fixed value, such as 0.95 . Figure 7 shows the movement of the boundary layer based on this criterion for the metallic fuel example with $L / R=6.4$. Examination of Figs. 6 and 7 shows how the boundary layer penetrates to the bottom of the short tube during the transition from elastic to viscous response. The rapid acceleration of the penetration rate is due to the strong exponential temperature dependence of the creep law.

Several additional finite element calculations (Case L1 and L2) were also performed to aid in the development of the analytical approximations given in the following section. In all of these calculations the cladding was made elastically very stiff by artificially increasing the cladding modulus as given in Table 1. Increasing the stiffness of the cladding this way did not significantly change the amount of fuel extrusion. However, it did simplify the interpretation of the finite element calculations for use in developing the boundary layer equations in the next section. For cladding much stiffer than the fuel the generalized plane strain solution for the initial elastic response of the fuel far from the free surface reduces to

$\varepsilon_{z}=\alpha_{C} \Delta T$,

$\sigma_{r}=\sigma_{\theta}=\sigma_{z}=-3\left(\alpha_{\mathrm{F}}-\alpha_{\mathrm{C}}\right) K_{\mathrm{F}} \Delta T$

Since the sress is already hydrostatic at any axial elevation, no local stress relexation occurs. This eliminates step 2 of the sequence for extrusion outlined above. However, because the hydrostatic stress state in Eq. (12) is approximately equal to the relaxed stress state of non-stiff cladding, the other steps of the sequence are the same giving nearly equal boundary layer penetration distances and total amounts of extrusion. 


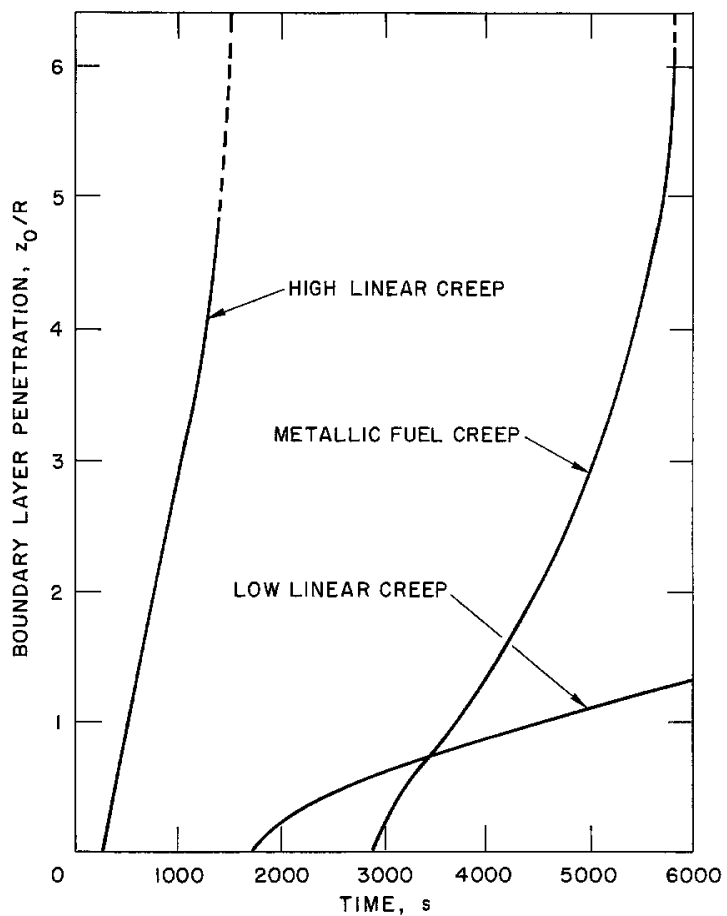

Fig.7. Boundary layer penetration distances for viscoelastic fuel slugs. The curve labeled "Metallic Fuel Creep" uses the non-linear fuel creep equation (Case F2 of Table 1). The curve labeled "High Linear Creep" uses a lower bound constant viscosity approximation to fuel creep (Case L1 and Table 1). The curve labeled "Low Linear Creep" uses an upper bound constant viscosity approximation to fuel creep (Case L2 of Table 2)

Figure 7 also shows the depth of boundary layer penetration for cases L1 and L2. Constant viscosities (with $n=1$ ) were used to give creep rates bounding the scale of the creep rate of metallic fuel. The 'low linear creep' case (L2) can be seen to result in a boundary layer penetration of only several radii. On the other hand, the 'high linear creep' case (L1) was sufficiently rapid so that the boundary layer reached the last row of elements well before the end of the simulated heating transient.

\section{Approximate boundary layer solution}

At large distances $z$ sufficiently far from the free surface the solution to the thermal extrusion of a viscoelastic material also obeys the generalized plane strain assumption. Even this solution normally requires numerical methods for the case of nuclear fuel pin behavior where radial temperature gradients exist and where the material behavior is highly non-linear. However, under conditions where the material is capable of flowing axially it must also be capable of deforming plastically at a sufficiently rapid rate to relax the normal stresses to a nearly uniform hydrostatic pressure across the radius. Since the cladding behaves thermo-elastically and since under generalized plane strain the fuel does not deform relative to the cladding, the resulting pressure is determined by the fuel compressibility and the cladding compliance. The rate of change of the fuel volume must equal the rate of change of volume inside a cladding segment. As discussed in the previous section, the cladding compliance does not significantly change the extrusion behaviour so that only the cladding volume change due to thermal expansion will be considered here in the development of the approximate boundary layer equations. Therefore, from Eq. (6),

$\frac{1}{v} \frac{\mathrm{d} v}{\mathrm{~d} t}=3 \alpha_{\mathrm{c}} \dot{T}=-\frac{\dot{P}}{K_{\mathrm{F}}}+3 \alpha_{\mathrm{F}} \dot{T}$

or, simply

$$
P(t)=P_{2} \frac{t}{t_{1}}
$$


for large $z$, where

$P_{2}=3 K_{\mathrm{F}}\left(\alpha_{\mathrm{F}}-\alpha_{\mathrm{c}}\right) \Delta T$

is the maximum pressure generated due to the total temperature rise $\Delta T$ over time $t_{1}=\Delta T / \dot{T}$.

The simple generalized plane strain solution given by Eq. (14) approximately satisfies all of the equations for the stated problem except the boundary condition $P=0$ at $z=0$. Near $z=0$ large pressure gradients develop so that relative axial flow of the slug inside the cladding tube takes place. In this zone it is reasonable to expect the following lubrication hypotheses to be valid

$\frac{\partial P}{\partial r} \ll \frac{\partial P}{\partial z} ; \quad \frac{\partial v_{z}}{\partial r} \gg \frac{\partial v_{z}}{\partial z}, \quad \frac{\partial v_{r}}{\partial r}, \quad \frac{\partial v_{r}}{\partial z} ;$

and inertia and compressibility effects are negligible compared to viscous effects.

The balance of momentum in the $z$-direction then reduces to

$\frac{\partial P}{\partial z}=\frac{2}{r} \sigma_{r z}$

and the continuity equation is given by

$\frac{\partial}{\partial t}(\varrho A)+\frac{\partial}{\partial z}\left(\varrho A \hat{v}_{z}\right)=0$

where $A$ is the cross-sectional area of the expanding tube and $\hat{v}_{z}$ is the radially averaged velocity. Substituting Eqs. (8-10) into Eq. (17) and integration across the radius gives

$\hat{v}_{z}(z, t)=-\alpha_{\mathrm{c}} \dot{T}(L-z)-\frac{C}{(n+3)} \frac{3^{(n+1) / 2}}{2^{n}} R^{n+1}\left(\frac{\partial P}{\partial z}\right)^{n}$

where we have made use of the boundary condition at $r=R$ that the fuel slug is bonded to the cladding tube. Except for the first term on the right-hand side, this equation is the same as the equation relating the average velocity to the pressure gradient for Poiseuille flow of a power-law fluid (Metzner 1961).

The density $\varrho$ is related to the pressure through Eq. (6) which can be written as

$\frac{1}{v} \frac{\mathrm{d} v}{\mathrm{~d} t}=-\frac{1}{\varrho} \frac{\mathrm{d} \varrho}{\mathrm{d} t}=-\frac{\dot{P}}{K_{\mathrm{F}}}+3 \alpha_{\mathrm{F}} \dot{T}:$

If, as assumed, the compressibility $\kappa=1 / K_{\mathrm{F}}$ is negligible in determining the flow velocity, substitution of Eq. (20) and the equation for the thermal expansion of the cladding,

$\frac{1}{A} \frac{\mathrm{d} A}{\mathrm{~d} t}=2 \alpha_{\mathrm{C}} \dot{T}$

into Eq. (18) gives

$\frac{\partial \hat{v}_{z}}{\partial z}=\left(3 \alpha_{\mathrm{F}}-2 \alpha_{\mathrm{C}}\right) \dot{T}$.

We now define the inner region over which viscous flow is significant as extending from $z=0$ to $z=z_{0}(t)$. At $z=z_{0}$ the inner solution is matched to the generalized plane strain outer solution by equating the pressure and the average velocity. Integration of Eqs. (22) and (19) then gives, for $0 \geq z \geq z_{0}$,

$\hat{v}_{z}=-\dot{T}\left(z_{0}-z\right)\left[3 \alpha_{\mathrm{F}}-2 \alpha_{\mathrm{C}}\right]-\alpha_{\mathrm{C}} \dot{T}\left(L-z_{0}\right)$

and

$P=\frac{n}{n+1} P_{1}\left(\frac{z_{0}}{R}\right)^{(n+1) / n}\left[1-\left(1-\frac{z}{z_{0}}\right)^{(n+1) / n}\right]$, 
where

$P_{1}=\left[\frac{3\left(\alpha_{\mathrm{F}}-\alpha_{\mathrm{C}}\right) \dot{T} 2^{n}(n+3)}{3^{(n+1) / 2} \mathrm{C}}\right]^{1 / n}$.

The boundary layer penetration distance $z_{0}$ can be found by equating the pressure at $z=z_{0}$ as given by Eqs. (14) and (24). The result is

$\frac{z_{0}}{R}=\left(\frac{n+1}{n} \frac{P_{2}}{P_{1}} \frac{t}{t_{1}}\right)^{n /(n+1)}$.

For the case of constant viscosity $(n=1,1 / \eta=3 C)$ Eq. (26) reduces to

$z_{0} / R=\left[K_{\mathrm{F}} / 4 \eta\right]^{1 / 2}$.

In order to illustrate the comparison between the approximate boundary layer solution given above with the previous finite element calculations we have calculated the boundary layer penetration distance and the axial displacement at the centerline of the free surface for case L1 of Table 1. The penetration distance for the finite element calculations was shown by the curve labeled low linear creep' in Fig. 7. Recall that this distance was defined for the finite element calculations by the axial position above which the ratio of the axial displacement of the fuel slug at the tube wall to the axial displacement at the centerline was less than 0.95 . Other choices for the ratio produce the curves shown in Fig. 8 which tend in the limit to the expected behaviour that $z_{0}=0$ at $t=0$. However, the finite size of the spatial grid restricts the resolution to which $z_{0}$ can be defined by this limit. Also shown in Fig. 8 by the dashed curve is the analytical boundary layer penetration distance as given by Eq. (27): The agreement with the extrapolated limit of the numerical results appears to be very good.

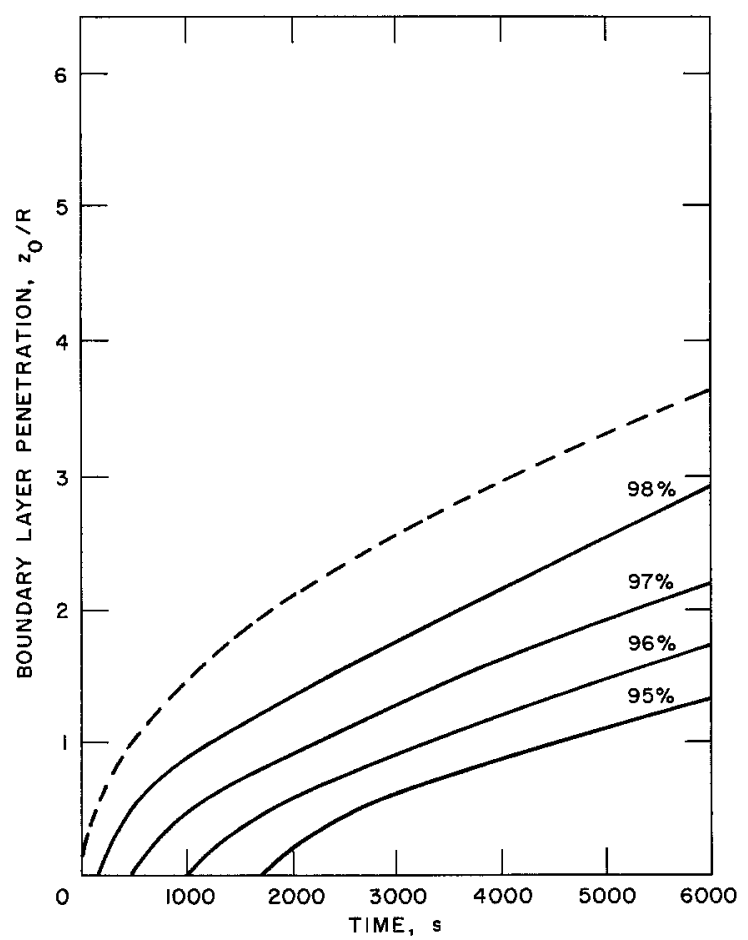

8

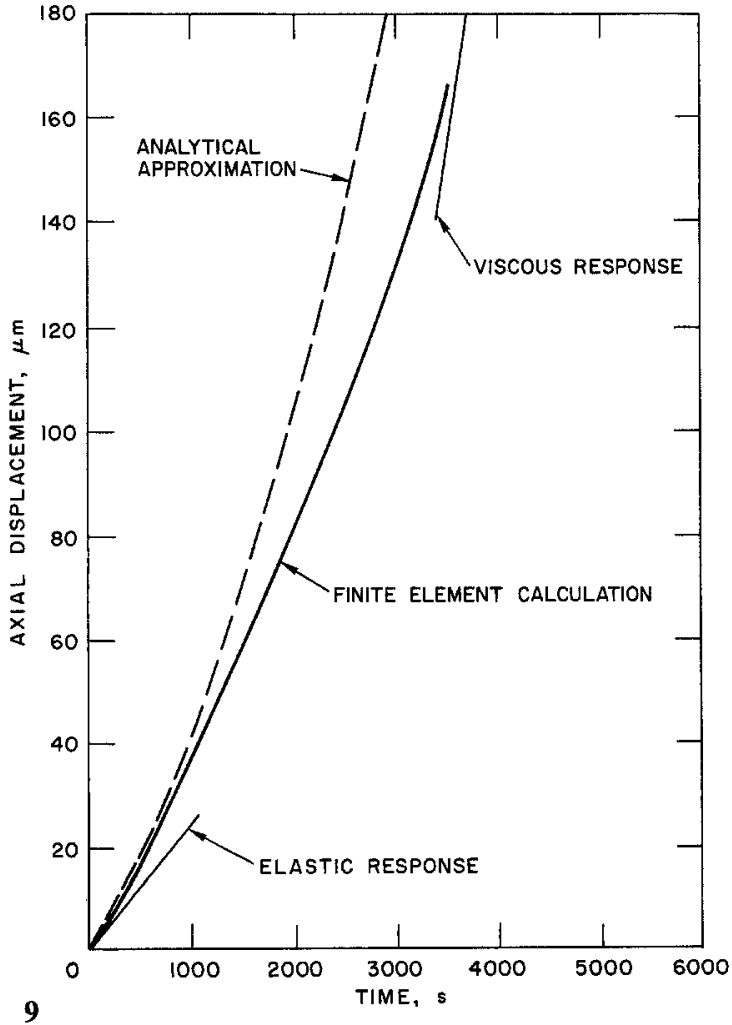

9

Figs. 8 and 9.8 Boundary layer penetration distance for low linear creep case L1 using various definitions of the boundary. The solid curves are for the finite element calculations while the dashed curve gives the analytical approximation. 9 Magnitude of axial displacement at the centerline of the free surface when thermal expansion is augmented by gas bubble expansion (Case $\mathrm{S} 1$ of Table 1). The solid curve is for the finite element calculations while the dashed curve gives the analytical approximations 
The boundary layer solution for the displacement at the centerline of the upper interface can be calculated by integrating the analytical expression for the velocity $v_{z}(r, z, t)$ over time. This velocity can easily be shown to be related to the average velocity $\hat{v}_{z}(z, t)$ at the surface by

$v_{z}(0,0, t)=\left(\frac{n+3}{n+1}\right)\left[\hat{v}_{z}(0, t)+\alpha_{\mathrm{c}} \dot{T} L\right]-\alpha_{\mathrm{e}} \dot{T} L$.

The resulting displacement for case L1 closely follows the finite element calculations with the final displacement at $6000 \mathrm{~s}$ equal to $170 \mu \mathrm{m}$ as compared to the numerical result of $160 \mu \mathrm{m}$. Comparison of axial displacements for the case of non-linear fuel creep (F2 in Table 1) gave even better agreement with the analytical calculation for the displacement at $6000 \mathrm{~s}$ equal to $167 \mu \mathrm{m}$ compared to the numerical result of $175 \mu \mathrm{m}$.

The calculations of extrusion driven by gas bubble expansion (discussed in the Appendix) show the same general behavior as simple thermal expansion. The extrusion is again limited to a boundary layer near the free surface at $z=0$. The analytical expressions for boundary layer penetration and axial displacement provide acceptable approximations to the much more complex calculations. We illustrate this behavior here by choosing the case labeled S1 in Table 1 where the bubble size is such that the effective fuel expansion coefficient is twice the linear expansion coefficient of the cladding. Figure 9 shows an example of the comparison of the axial displacement at the free surface as calculated by the finite element method and by the analytical boundary layer approximation. Although the shape of the curves and two asymptotes for the velocity at this surface are the same, it appears that the analytical expression underestimates somewhat the time over which the transition occurs.

\section{Axial extrusion of metallic reactor fuel during overheating transients}

In the preceding sections we have shown that the simple boundary layer equations give reasonable agreement with two-dimensional finite element calculations for extrusion under conditions relevant to the transient expansion of metallic reactor fuel. Comparisons of calculations were provided for fairly short tubes $(L / R<6.4)$ so that the numerical calculations would not be prohibitively time consuming. Here we wish to extend the analytical results to full length reactor fuel designs with $L / R=295$ and $R=3.10 \mathrm{~mm}$. We will also consider higher heating rates in the range of $10-100 \mathrm{~K} / \mathrm{s}$ which are more typical of reactor accident conditions.

Table 2 gives the calculated boundary layer penetration distance $z_{0} / R$ and the centerline displacement at the free surface $u_{b}$ for metallic fuel extrusion at various heating rates. Also provided is the displacement $u_{\mathrm{gps}}$ that would result if the generalized plane strain assumption were valid along the entire length. Fuel creep was assumed to be given by the non-linear Eq. (5) and the extrusion potential was increased by augmenting the fuel thermal expansion to account for transient fission gas swelling. It can be seen from the table that the extent of the extrusion zone is only about 15 times the radius (or $5 \%$ of the fuel length) by the time of fuel melting when the fuel is heated at $10 \mathrm{~K} / \mathrm{s}$. The axial displacement at the top of the fuel $u_{b}$ is about $8.5 \%$ larger than would be calculated using the generalized plane strain assumption. However, this additional expansion comes from a small zone near the top of the pin so that it would not add significantly to the total fuel reactivity feedback.

Table 2. Maximum boundary layer penetration and displacements for full length tubes ${ }^{a}$ with thermal expansion augmented by gas expansion ${ }^{\mathbf{b}}$

\begin{tabular}{rcll}
\hline$\dot{T}, \mathrm{~K} / \mathrm{s}$ & $z_{0} / R$ & $u_{\mathrm{b}}, \mathrm{mm}$ & $u_{\mathrm{gps}}, \mathrm{mm}$ \\
\hline 0.1 & 47 & 13.3 & 10.6 \\
1.0 & 27 & 12.1 & 10.6 \\
10.0 & 15 & 11.5 & 10.6 \\
100.0 & 8 & 11.1 & 10.6 \\
\hline
\end{tabular}

${ }^{\mathrm{a}} L / R=295, R=3.10 \mathrm{~mm} ;{ }^{\mathrm{b}} \alpha_{\mathrm{F}}($ effective $)=3.9 \times 10^{-5} \mathrm{~K}^{-1}$ 
In the above calculations the only source of fuel compressibility was due to the elastic compressibility of the fuel matrix. If fission gas bubbles are free to expand they also must be free to compress. The results in Table A- 1 of the Appendix suggest that for $0.1 \mu$ radius bubbles the fuel compressibility can be as high as $7.5 \times 10^{-3} \mathrm{MPa}^{-1}$. For the $10 \mathrm{~K} / \mathrm{s}$ heating example in Table 2 the higher compressibility would further reduce the boundary layer penetration to $z_{0} / R=0.65$. Thus within the range of reasonable assumptions for the fuel expansion and the fuel compressibility, solid fuel extrusion is limited to a small zone near the top of the fuel less than about 10 radii in length.

One of the major factors that limits solid extrusion is the high effective viscosity. We have already noted in this regard that the effective viscosity of solid uranium-based alloys is some 10-15 orders of magnitude greater than the viscosity of liquid uranium. It is obvious from the analysis given previously that if bulk melting occurs during a reactor transient there will be little viscous resistance to subsequent extrusion of liquid fuel. Significant extrusion of fuel could also occur prior to bulk melting if there is local melting at the fuel-cladding interface. Although interdiffusion metallurgically bonds uranium fuel alloys to iron-based cladding alloys after serveral percent burnup, some of the phases present at the interface have solidus temperatures as low as $1000-1100 \mathrm{~K}$. The large decrease in viscosity upon melting at the interface would likely release the remaining solid fuel so that it can extrude axially. The resulting motion of the solid slug can be determined by separate generalized plane strain analyses of the fuel and cladding deformation.

\section{Conclusions}

Axial extrusion of an expanding non-linear viscoelastic solid inside a long cylindrical tube has been analyzed. Both differential thermal expansion and expansion of gas bubbles in the solid have been considered as driving forces. In both cases finite element calculations have shown that the following sequence of events leads to extrusion of the solid relative to the tube:1) the initial response is nearly elastic and can be determined by generalized plane strain calculations; 2 ) as creep begins the stresses at any axial elevation relax to a hydrostatic state, again through plastic deformation; 3 ) the resulting axial pressure gradients are small except near the free surface where shear deformation begins; 4 ) as the shear deformation relieves the pressure gradient, a boundary layer separating flowing material from the rest of the slug moves downward until it reaches the bottom of the cylinder; 5) when the boundary layer reaches the bottom of the cylinder the entire slug flows like a non-Newtonian fluid at a constant rate determined by the expansion rates of the slug and its cylinder.

A simple boundary layer analysis has been developed to provide analytical expressions to describe the above extrusion process. At distances far from the free surface the generalized plane strain analysis shows that the nearly hydrostatic state of stress is governed by the material compressibilities. Near the free surface at the top of the cylinder the flow can be determined by applying lubrication theory hypotheses. The boundary between the zones can then be calculated by matching the pressure and average velocity from these inner and outer solutions. For linear viscoelastic materials the growth of the boundary layer is proportional to $\sqrt{K t / \eta}$ where $t$ is the time, $K$ is the effective bulk modulus and $\eta$ is the effective viscosity. Comparisons of calculations based on the approximations with finite element results show good agreement for extrusion of both linear and non-linear viscoelastic materials.

The above analysis has been applied to investigate axial extrusion of metallic fast reactor fuel during overheating events. Such extrusion has been postulated to provide a passive negative reactivity source which could contribute to reactor shutdown during an overheating event. However, our calculations indicate that there will be little relative axial motion between solid fuel and the cladding as long as the fuel and cladding are locked together at the fuel-cladding interface. Because of the small pressure gradients, large compressibilities, and large viscosities involved, any extrusion would be limited to a zone near the top of the fuel of about 10 radii in length for a $10 \mathrm{~K} / \mathrm{s}$ heating ramp. Of course even if there is no extrusion, simultaneous expansion of the fuel and cladding contributes to the negative reactivity feedback. Furthermore, relative extrusion can occur if there is bulk melting of the fuel or if there is local melting of uranium-iron eutectic phases that have formed at the fuel-cladding interface during irradiation. 


\section{Appendix}

In this section we consider augmentation of thermal expansion of the viscoelastic slug by the expansion of very small $(r<0.1 \mu \mathrm{m})$ gas bubbles trapped in the solid. For our application to metallic reactor fuel we note that experience with EBR-II driver fuel has shown that metallic fuel pins may retain an average amount of fission gas equal to seven times the original fuel volume if this gas were expanded to one atmosphere at room temperature (STP) (Walters, Seidel and Kittel 1984). During heating transients mechanisms exist [such as grain boundary diffusion (Gruber and Kramer 1986)] by which these bubbles can rapidly expand in response to increases in the gas pressure.

We assume here that the gas bubbles in the slug are always in pressure equilibrium. The pressure $P_{\mathrm{g}}$ of the gas inside the bubbles is therefore

$P+2 \gamma / r_{\mathrm{b}}=P_{\mathrm{g}}$,

where $r_{\mathrm{b}}$ is the bubble radius, $P$ is the hydrostatic pressure in the surrounding material and $\gamma$ is the surface tension. The gas pressure is related to the bubble volume $V_{\mathrm{b}}$ by the ideal gas law

$P_{\mathrm{g}} V_{\mathrm{b}}=P_{\mathrm{g}} \frac{4}{3} \pi r_{\mathrm{b}}^{3}=m k T$,

where $m$ is the number of gas atoms per bubble and $k$ is Boltzman's constant. We have also assumed for the purposes of this paper that all of the bubbles are of the same size, there is no coalescence and the bubbles are sufficiently small so that

$|P| \ll 2 \gamma / r_{\mathrm{b}}$.

Differentiating Eqs. (A.1) and (A.2) and substituting Eq. (A.3) then gives the following linearized approximation to the transient fuel swelling $e_{m b}$ due to fission gas bubble expansion

$e_{m \mathrm{~b}}=V_{\mathrm{p}} \frac{\Delta V_{\mathrm{b}}}{V_{\mathrm{b}}}=V_{\mathrm{p}}\left[\frac{3}{2} \frac{\Delta T}{T}-\frac{3}{4} \frac{r_{\mathrm{b}}}{\gamma} \Delta P\right]$,

where $V_{\mathrm{p}}$ is the volume fraction of bubbles (porosity).

As shown in Table A.1, all of the factors in the above equations can be determined for metallic fuel from the total gas content of the fuel and the bubble radius. Also shown in this table are the thermal expansion and elastic compressibility of the fuel matrix as defined by Eq. (6) and Table A.1.

The most straightforward way to estimate the total transient swelling $e_{m}$ is to simply add $e_{m}$ from Eq. (6) and $e_{m b}$ from Eq. (A.4). Combining the coefficients of the terms involving $\dot{T}(\Delta T / \Delta t$ ) gives an effective linear expansion coefficient

$\alpha_{\mathrm{F}}(\mathrm{effective})=\alpha_{\mathrm{F}}+V_{\mathrm{p}} / 2 T$

Similarly, the effective bulk modulus is given by the sum of the terms multiplying $\dot{\sigma}_{m}(-\Delta P / \Delta t)$, or $1 / K_{\mathrm{F}}($ effective $)=1 / K_{\mathrm{F}}+3 V_{\mathrm{p}} r_{\mathrm{b}} / 4 \gamma$.

Calculation of the effective fuel expansion coefficient and the effective compressibility using Eqs. (A.5) and (A.6) and the bubble parameters given in Table A.1 indicates that bubble expansion will dominate elastic expansion for bubbles with radii greater than about $0.01 \mu \mathrm{m}$

Table A.1. Bubble swelling factors in metallic reactor fuel for different bubble sizes

\begin{tabular}{lclllll}
\hline$r_{\mathrm{b}}, \mu \mathrm{m}$ & $2 \gamma / r_{\mathrm{b}}, \mathrm{MPa}$ & $V_{\mathrm{p}}$ & $V_{\mathrm{p}} / 2 T, K^{-1}$ & $\alpha_{\mathrm{F}}, K^{-1}$ & $3 V_{\mathrm{p}} r_{\mathrm{b}} / 4 \gamma, \mathrm{MPa}^{-1}$ & $1 / K_{\mathrm{F}}, \mathrm{MPa}^{-1}$ \\
\hline 0.1 & 20 & 0.1 & $6.5 \times 10^{-5}$ & $2.1 \times 10^{-5}$ & $7.5 \times 10^{-3}$ & $1.15 \times 10^{-4}$ \\
0.01 & 200 & 0.01 & $6.5 \times 10^{-6}$ & $2.1 \times 10^{-5}$ & $7.5 \times 10^{-5}$ & $1.15 \times 10^{-4}$ \\
0.001 & 2000 & 0.001 & $6.5 \times 10^{-7}$ & $2.1 \times 10^{-5}$ & $7.5 \times 10^{-7}$ & $1.15 \times 10^{-4}$ \\
\hline
\end{tabular}

Assuming: 1) $7 \mathrm{~mm}^{3}$ gas $\times$ STP per $\mathrm{mm}^{3}$ fuel; 2) $T=773 \mathrm{~K}$, 3) $\gamma=1 \mathrm{~J} / \mathrm{m}$ 


\section{Acknowledgements}

J. D. Demaree's work was supported by the Argonne Division of Educational Programs through a Student Research Participation Program Grant. The authors wish to thank J. M. Kennedy for providing the STRAW code used for the finite element calculations, R. H. Sevy for discussion with regards to the equilibrium fission gas swelling model, and K. S. Rank and L. J. Miller for preparing the manuscript.

\section{References}

Cahalan, J; ; Sevy, R.; Su, S. (1985): Accomodation of unprotected accidents by inherent safety design features in metallic and oxide-fueled LMFBRs. In: Proc. of the international topical meeting on fast reactor Safety, Knoxville, TN, April 21-25, 1985, CONF-850410, Vol. 1, pp. 29-33. LaGrange Park, IL: American Nuclear Society

Gatewood, B. (1957): Thermal stresses, p. 210. New York: McGraw-Hill

Gruber, E.; Kramer, J. (1986): Gas bubble growth mechanisms in the analysis of metal fuel swelling. In: Proc. of the ASTM Symp. on the effects of radiation on materials, Seattle, WA, June 23-25, 1986, Philadelphia, PA : ASTM (in preparation) Matthews, J. (1970): Thermal stress in a finite heat generating cylinder. Nucl. Eng. Des. 12, 291-296

Metzner, A. (1961): Flow of non-Newtonian fluids. In: Streeter, V. (ed): Handbook of fluid dynamics, pp. 7-4 to 7-17. New York: McGraw-Hill

Miles, K.Jr.; Kalimullah (1985): The inherent safety phenomenon of fission-gas induced axial extrusion in oxide and metal fueled LMFBRs. In: Proc. of the international topical meeting on fast reactor safety, Knoxville, TN, April 21-25, 1985, CONF-850410, Vol. 1, pp. 103-110. LaGrange Park, IL: American Nuclear Society

Ofte, D. (1967): The viscosities of liquid uranium, gold and lead. J. Nucl. Mater., 22, 28-32

Schreyer, H.; Kennedy, J.; Schoeberle, D. (1983): Thermoviscoplastic analysis of first walls subjected to fusion power transients. Trans. ASME J. Pressure Vessel Tech. 105, 42-51

Toupin, R. (1965): St. Venant's principle. Arch. Ratl. Mech. Anal. 18, 83-92

Valentin, R; ; Carey, J. (1979): Thermal stresses and displacements in finite, heat-generating circular cylinders. Nucl. Eng. Des. 12, 277-290

Walters, L.; Seidel, B.; Kittel, J.H. (1984): Performance of metallic fuels and blankets in liquid-metal fast breeder reactors. Nucl. Technol. 65, 179-231

Communicated by S.N. Atluri, August 6, 1986 